CAHIERS DE

NARRATOLOGIE

\section{Cahiers de Narratologie}

Analyse et théorie narratives

36 | 2019

Rhétorique et représentations de la culture mafieuse. Images, rituels, mythes et symboles

\title{
Les rouages d'un débat. Réponse à Ilias Yocaris
}

\section{Raphaël Baroni}

\section{(2) OpenEdition \\ Journals}

Electronic version

URL: http://journals.openedition.org/narratologie/10134

ISSN: $1765-307 X$

Publisher

LIRCES

\section{Electronic reference}

Raphaël Baroni, «Les rouages d'un débat. Réponse à llias Yocaris », Cahiers de Narratologie [Online],

36 | 2019, Online since 20 December 2019, connection on 21 December 2019. URL : http:// journals.openedition.org/narratologie/10134

This text was automatically generated on 21 December 2019.

Article L.111-1 du Code de la propriété intellectuelle. 


\title{
Les rouages d'un débat. Réponse à Ilias Yocaris
}

\author{
Raphaël Baroni
}

\section{Critiques et résistances constructives}

1 J'espère que l'on me croira sincère si j'affirme que j'ai été très stimulé par la lecture que propose Ilias Yocaris de mon dernier livre. Si les critiques favorables offrent le sentiment confortable de se voir récompensé de ses efforts, celles qui sont nuancées, voire plus nettement négatives (c'est-à-dire les critiques réellement critiques), sont indéniablement les plus fécondes pour faire avancer la pensée, pour prendre conscience des angles-morts de son argumentation et des efforts supplémentaires qu'il s'agit de fournir pour surmonter d'éventuels malentendus. C'est donc la seconde partie du compte-rendu d'Ilias Yocaris qui retiendra surtout mon attention et je suis également reconnaissant à la rédaction des Cahiers de narratologie de me donner un droit de réponse, de manière à apporter quelques éclaircissements, cette extension n'étant pas dépourvue, on le verra, de repentirs.

\section{Pour un dépassement des dichotomies}

2 Je commencerai par tenter de répondre aux critiques formulées à l'encontre du deuxième chapitre, qui visait à réhabiliter la lecture pour l'intrigue, ou plus exactement à justifier l'intérêt qu'il peut y avoir à essayer d'en démonter les mécanismes textuels, en classe ou ailleurs. À cet endroit, le commentaire de mon lecteur, jusque-là plutôt bienveillant, se fait plus tranchant, voire même franchement sarcastique. Ilias Yocaris affirme en effet que l'ouvrage se voit soudain «plombé par un cortège assez invraisemblable d'approximations, de contre-vérités et d'affirmations discutables à l'emporte-pièce qui renvoient aux pires poncifs du pédagogisme scolaire ».

3 Il me faut reconnaître que cette partie pose problème si elle est lue comme la mise en cause de pratiques liées à l'enseignement de la littérature, qui doivent être à peu près 
aussi diverses qu'il y a d'enseignante's sur cette planète. À me relire, j'avoue que certains passages (notamment en page 49) pourraient en effet laisser croire que je cible les études littéraires sur la base de stéréotypes discutables. Dans cette partie, j'ai probablement tort de rappeler que Daniel Pennac dépeignait l'institution scolaire comme une école du « désenchantement ». Il est possible que ce soit l'expérience vécue par cet écrivain, mais rien ne permet d'affirmer que les cours de littérature s'apparentent toujours à une telle désillusion, et encore moins que ce problème soit lié à l'usage de paradigmes formalistes, ainsi que le suggère par exemple Todorov (2007). À vrai dire, si je me fonde sur mon propre vécu, je n'ai que des souvenirs positifs de mes cours de français, que ce soit au collège (je me souviendrai toujours des cours extraordinaires du regretté Jean-Luc Sahy), au lycée (où je me suis réorienté des sciences vers les humanités) ou à l'Université, ce qui explique mon orientation professionnelle actuelle. Mentionner Pennac visait surtout à rappeler que dans le contexte actuel, marqué par une crise des études littéraires ${ }^{1}$, il est essentiel de réfléchir aux moyens de redonner goût à cette discipline, notamment en réfléchissant à ses objectifs et à ses moyens de formation.

4 En ce qui concerne la réalité du terrain, à part le contexte académique lausannois, dans lequel j'évolue professionnellement depuis une quinzaine d'années (puisque les chercheurs enseignent également et, dans mon cas, à des lecteurs alloglottes de différents niveaux), je ne peux que confesser une ignorance à peu près totale de la diversité des contextes liés à l'école obligatoire et post-obligatoire en France, ou même ailleurs en Suisse romande. Tout au plus puis-je renvoyer à quelques études récentes (Gabathuler 2016; Ronveaux et al. 2019), qui dressent d'ailleurs un tableau beaucoup plus nuancé de la manière dont les valeurs éthiques et esthétiques des textes sont discutées en classes et s'intègrent dans les activités de commentaire des œuvres.

5 La simple question de connaître la place que tient aujourd'hui la théorie du récit dans les différents niveaux de la scolarité, et la manière dont elle est incorporée (ou non) à des lectures concrètes et contextualisées, est en soi une question passionnante, mais à laquelle il est pour le moment difficile de répondre. Il me semble néanmoins réducteur d'affirmer, à l'instar d'Ilias Yocaris, que «l'expérience du terrain montre hélas que l'enseignement secondaire en France se trouve actuellement à des années-lumière de toute approche formaliste des œuvres littéraires: on privilégie au contraire un thématisme des plus réducteurs ». Si Yocaris se fonde sur une expérience documentée, je serais heureux d'en connaître les sources. Et j'ajouterais que certaines approches thématiques peuvent être parfaitement valables si elles constituent un point de départ pour discuter les enjeux éthiques liés aux intrigues littéraires.

6 En attendant, pour y voir plus clair, je suis en train de préparer une demande de financement auprès du Fonds national suisse pour la recherche scientifique dans le but de constituer une équipe dont l'objectif serait précisément d'investiguer sur le terrain, à l'aide de questionnaires et d'entretiens, les difficultés auxquelles sont confrontés les enseignante's, ainsi que les solutions qu'ils ou elles ont élaborées pour les surmonter. La lecture des plans d'étude romands et les premiers questionnaires qui nous ont été retournés montrent que l'apprentissage de compétences théoriques demeure un objectif central et une pratique courante dans le secondaire I et II en Suisse romande, ce qui est évidemment réjouissant. Mon objectif, en tant que narratologue, n'a évidemment jamais été de rejeter les approches formalistes, mais bien d'en remotiver l'usage en offrant de nouveaux leviers pour l'interprétation des textes. Le fait même 
que je doive expliquer cela me semble assez incroyable, mais la lecture que propose Ilias Yocaris de mon deuxième chapitre m'y contraint.

7 Le problème n'est pas la théorie, mais son figement dans un paradigme structuraliste, présenté comme la seule approche praticable pour l'analyse textuelle. Catégoriser un narrateur d'extra-hétérodiégétique et reconstruire le schéma quinaire d'une œuvre me semblent des activités relativement pauvres si on ne s'intéresse pas à la manière dont ces paramètres influent sur l'intérêt de l'histoire, sur son sens ou son interprétation. Si l'on accepte de ne pas considérer la narratologie comme un simple moment de l'histoire littéraire, mais comme une discipline évolutive et traversée par des paradigmes concurrents, il me semble que cette approche, qui intègre forcément une certaine dose de formalisme, a beaucoup mieux à offrir que les quelques outils figés par les ouvrages de synthèse et par les manuels scolaires que j'ai pu consulter (voir Baroni 2020). Cette théorie du récit contemporaine a notamment été largement enrichie par l'apport de la stylistique, de la linguistique textuelle (Adam 2005 ; Rabatel 2009) et de l'analyse du discours (Maingueneau 2004), mais aussi par la rhétorique et les approches cognitivistes, ainsi que je ne cesse de le répéter depuis de nombreuses années.

Quoi qu'il en soit, pour revenir sur ces "stéréotypes", qu'llias Yocaris rattache fort justement «à ce qu'on pourrait appeler le folklore pédagogique ", j'aimerais ajouter à son crédit que dans son compte-rendu, Bertrand Daunay formule lui aussi une critique de ces représentations dichotomiques de la lecture :

Peut-être pourrait-on aussi s'interroger sur le choix de reprendre au début du chapitre 2 (sous le titre "Premier et second degrés de la lecture ») les dichotomies classiques avancées pour classer les modalités (scolaires ou non) de lecture. Que ces dichotomies aient été et soient encore reprises par des didacticiens (dont certains sont cités ici), n'empêche pas qu'elles reposent sur une fable - que j'avais, sans grand succès, tenté de déconstruire il y a vingt ans (Daunay 1999), non pour laisser accroire que ces modalités «se rejoignent dans une conception "postmoderne" de la littérature » (pour reprendre les termes de Baroni, p. 50), mais tout simplement pour faire apparaitre leur inconsistance théorique. (Daunay 2019 : \$9)

9 Si ce chapitre pose quelques problèmes dans la mesure où il semble créditer de telles oppositions caricaturales, il me semble malgré tout utile de clarifier sa visée argumentative. La mention de ces dichotomies n'avait pas pour but de critiquer les pratiques de lecture savantes, et encore moins de mettre en cause les pratiques interprétatives encouragées par le contexte scolaire. Il s'agissait plutôt d'exposer ces "stéréotypes " pour montrer que de telles représentations ont conduit à un désintérêt pour les questions relatives à l'intrigue et à l'immersion fictionnelle, surtout du côté des théoriciens du récit. Si la distinction entre le premier et le second degré de la lecture est largement illusoire, elle n'en demeure donc pas moins constitutive de nombreuses représentations qui ont longtemps servi à dévaloriser certains rapports au texte pour en privilégier d'autres.

De toute évidence, Ilias Yocaris n'a pas saisi l'ironie des propos de Jérôme David, qui n'a jamais prétendu que la lecture au premier degré ne serait que «l'apanage des femmes, des enfants et des pauvres». Il affirme au contraire que tout le monde, femmes et hommes, enfants et adultes, lecteurs occasionnels et lecteurs professionnels, lit au premier et au second degré. Mais la généralisation de l'accès à la lecture que l'on observe au cours du XIXe siècle a conduit à des différenciations symboliques construisant des rapports aux textes jugés plus ou moins légitimes ou illégitimes. Son propos visait à montrer que certaines institutions de l'époque ont créé et véhiculé des 
représentations stigmatisant certains types de lecteurs (ou de lectrices) et certains modes d'appréhension des textes. Il s'agissait de créer des distinctions artificielles de manière à reléguer la lecture en immersion dans les limbes du bovarysme, un peu comme aujourd'hui certains considèrent les jeunes passionnés par les jeux vidéo comme des mass murderer en puissance.

Le fondement de ces dichotomies trompeuses se laisse facilement deviner : il réside dans l'opposition naturalisée entre l'art et l'argent, entre les procédés esthétiques et commerciaux, entre la littérature jugée "véritable » et la littérature «à vapeur », dont Bourdieu (1971) a montré qu'elle polarise le champ artistique depuis la marchandisation de la culture et son entrée dans l'ère médiatique et la production de masse. Pour ne mentionner que le domaine que je connais le mieux, on peut expliquer sur cette base le peu d'intérêt manifesté par les narratologues de la période structuraliste pour les questions relatives au suspense ou à l'immersion, car la plupart d'entre eux considéraient ces mécanismes comme relevant exclusivement de la paralittérature et comme menant à une aliénation du lecteur. Il a fallu attendre, entre autres, les travaux de Jean-Marie Schaeffer (1989) sur l'immersion fictionnelle, puis ceux de Vincent Jouve (1992) sur le personnage, pour observer un timide rééquilibrage des valeurs dans les discours dominants.

L'objectif de cette première partie de mon livre visait donc à clarifier ma définition de l'intrigue - à la fois pour en préciser la nature et pour en élargir la portée à n'importe quelle œuvre susceptible d'intriguer son lecteur, même Les Gommes d'Alain RobbeGrillet $^{2}$ ou Le Roi Cophetua de Julien Gracq. Il s'agissait ensuite de poursuivre dans la voie ouverte par Schaeffer et par Jouve en insistant sur l'importance anthropologique des phénomènes esthétiques fondés sur l'immersion et la consistance mimétique du monde et des personnages. Je voulais aussi montrer l'importance adaptative et éthique des intérêts liés au développement de l'intrigue et à la tension narrative. Une telle mise au point me semblait nécessaire car les travaux des narratologues "classiques», qui restent la base de la plupart des manuels et ouvrages de synthèse, avaient tendance à reléguer ces aspects au rang d'épiphénomènes caractéristiques de la littérature populaire ou commerciale, alors qu'ils sont au cœur de la fonction anthropologique que l'on peut associer aux récits que j'ai appelé mimétiques, c'est-à-dire les formes narratives immersives et intrigantes, qui constitue la grande majorité des récits de fiction.

13 Il n'y a donc aucun mépris ou aucune condescendance dans les propos de Jérôme David concernant les jeunes ou les femmes, ni dans les miens j'espère, puisque l'objectif était de dépasser ces oppositions réductrices. Dans ce chapitre, je pensais avoir été assez explicite sur ce point, puisque je commençais par rappeler que « nous assistons dans le champ de la didactique à un rééquilibrage entre différentes formes d'appropriation du texte littéraire » (Baroni 2017a : 51) et que je poursuivais en insistant sur «la nécessité de décloisonner les lectures de plaisir et les lectures critiques» (Baroni 2017a: 54). Je citais en particulier ma collègue Chiara Bemporad qui, sur la base de témoignages de lecteurs, a insisté sur l'importance de réviser ce qu'elle définit comme les «manières traditionnelles » de considérer la lecture (cité dans Baroni 2017a: 50). Elle insiste en particulier sur le fait que :

le plaisir joue un rôle clé pour briser ces oppositions. Une telle approche permet un décloisonnement des représentations, attitudes et actions qui ne peut être que bénéfique pour la didactique de la littérature (Bemporad 2007 : 80) 
Évidemment, il ne faut pas en rester au simple constat que la lecture scolaire devrait prendre en considération le plaisir esthétique. Chiara Bemporad rappelle ainsi que "valoriser différents modes de lecture " passe aussi par un élargissement des horizons esthétiques, ce qui passe en particulier par l'introduction d'outils d'analyse :

Parallèlement, l'enseignant devrait continuer de fournir aux lecteurs en formation des outils d'analyse pour qu'ils puissent avoir accès aux sens de textes de plus en plus complexes. C'est ainsi qu'en construisant de nouvelles compétences, on permettra de diversifier les modes de lecture, pour élargir la gamme des plaisirs littéraires. (Bemporad $2014: 81$ )

Un autre point crucial consiste à comprendre que la réhabilitation de la lecture immersive et intriguée que je défends ne vise nullement à se cantonner à un rapport spontané et intuitif au texte. De manière tout à fait explicite, j'affirme privilégier la voie d'une théorisation de la littérature qui permette de traiter ces aspects qui ont été longtemps négligés, et non de m'appuyer sur une expérience immédiate des lecteurs intrigués, que l'on pourrait par exemple saisir par le biais de journaux ou de témoignages spontanés. Je précise qu'il s'agit donc bien de développer des outils permettant de mieux comprendre le fonctionnement textuel de l'intrigue, et je terminais ce chapitre en affirmant d'ailleurs qu'il s'agissait :

de dépasser un rapport purement émotionnel ou subjectif au texte, tout en fournissant des arguments pour discuter de la valeur cognitive ou éthique des récits immersifs et intrigants. Même au bout de la lecture, ou dans ces intervalles critiques qui peuvent constituer les étapes intermédiaires d'une lecture suivie en classe, analyser comment le récit est parvenu à nous séduire répond alors à l'ambition légitime d'acquérir une plus grande autonomie face aux textes narratifs. (Baroni 2017a : 54).

\section{Une approche « intégralement axée sur la réception »?}

16 Le point que je viens d'évoquer mérite d'être développé, car il répond à une autre critique, très générale celle-là, et qui me semble cette fois-ci largement infondée. Yocaris affirme en effet que « l'approche de la mise en intrigue défendue par Baroni est intégralement axée sur la réception des récits fictifs, en faisant fi de leur (contexte de) production». Il en conclut que mon approche «minore par définition l'importance des paramètres liés à la stratégie discursive auctoriale dont la mise en intrigue est le fruit ».

17 J'ai été très surpris par cette affirmation, car l'approche que je propose dans ce livre diffère du modèle théorique développé dans La Tension narrative (2007) précisément par le souci de mettre l'accent sur les mécanismes textuels et les rouages narratifs de l'intrigue (d'où le titre de l'ouvrage). Ainsi que je le déclare dès l'introduction, il s'agissait d'aborder le phénomène sous un angle non pas cognitif, mais résolument rhétorique, c'est-à-dire de fournir des moyens pour une analyse formelle des dispositifs littéraires qui visent à intriguer le lecteur. Je vois mal comment on pourrait saisir cette « stratégie discursive auctoriale " autrement qu'en prêtant attention aux différents facteurs formels et linguistiques que je passe en revue dans la partie 2. Si cet inventaire est insuffisant aux yeux de Yocaris, je ne peux que l'encourager à le compléter lui-même, ainsi d'ailleurs que j'encourage à le faire l'ensemble de mes lecteurs qui travaillent dans le domaine de la théorisation de la littérature.

18 J'ai donc du mal à comprendre d'où peut venir l'affirmation selon laquelle mon approche serait « intégralement axée sur la réception »? Certes, j'insiste sur le fait qu'il 
me semble essentiel de définir le noud de l'intrigue non sur la base d'une analyse strictement interne et thématique - p. ex. le personnage rencontre une complication qui vient perturber une situation initiale - mais sur la base d'une analyse fonctionnelle du discours narratif: la complication que rencontre un personnage devient l'un des moyens (parmi de nombreux autres) qui permettent à l'auteur de nouer une intrigue en induisant du suspense dans la progression narrative. Toutefois, ainsi que je l'explique au début de la partie 2, ce passage du formalisme au fonctionnalisme ne vise nullement à faire l'impasse sur le premier aspect, mais il consiste à lier forme et fonction, ce qui exige simplement d'enrichir l'analyse traditionnelle de l'intrigue, fondée sur une lecture chronologique et thématique du parcours du protagoniste, en reconnaissant qu'il existe de nombreux moyens (thématiques, mais aussi textuels, stylistiques, etc.) pour créer ou renforcer la tension nouée par l'intrigue jusqu'à son éventuel dénouement.

L'idée n'était donc pas de prendre comme point de départ une appréhension intuitive ou subjective du texte, par exemple en se fondant sur des intuitions ou des témoignages de lecteurs, mais de se donner les moyens d'analyser les dispositifs textuels et stylistiques que les auteurs peuvent mobiliser pour nouer et dénouer leurs intrigues, c'est-à-dire pour créer des " arcs narratifs ", au sens que l'on donne à cette expression dans les discours sur la scénarisation des films ou des séries. Pour rendre compte de la dynamique de l'intrigue, l'analyse thématique doit être complétée par celle de la configuration temporelle du discours narratif, de la voix et du mode utilisés pour raconter les événements, et d'autres aspects plus directement linguistiques, comme le passage d'un imparfait à un passé simple, l'usage d'un adverbe temporel tel que "soudain ", ou l'utilisation d'hyperonymes aux référents provisoirement ambigus.

C'est là toute la difficulté de l'entreprise, qui justifie cette deuxième partie très longue et assez touffue: réconcilier l'analyse fonctionnelle de l'intrigue avec l'usage des typologies structuralistes et de l'analyse du discours littéraire, de manière à rendre possible un étayage aussi précis que possible de l'interprétation. L'intérêt éventuel de l'analyse des rouages de l'intrigue dépend donc de l'efficacité des outils qui peuvent être déployés dans le commentaire, et je reconnais volontiers, à l'instar d'Ilias Yocaris,

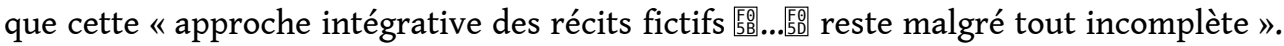
Toutefois, l'analyse de Derborence que je propose dans la partie 3 montre que l'étude formelle de la dynamique de l'intrigue n'est pas incompatible avec une réflexion contextuelle liée à la biographie de l'écrivain ou au contexte culturel dans lequel il écrit ses romans. Ce n'est simplement pas l'aspect sur lequel j'ai mis l'accent, ma perspective se situant davantage dans une perspective orientée sur les structures narratives.

21 Je reconnais cependant que l'on peut considérer comme regrettable de ne pas avoir intégré une analyse plus détaillée de la généricité des textes, de la scénographie ou du narrateur non fiable (au sens de Wayne C. Booth), dont dépendent souvent des effets de curiosité ou de surprise. J'aurais aussi pu aussi développer davantage l'analyse du cadre de l'action (décors, monde narratif) et celle du rôle que l'on peut attribuer aux séquences descriptives, dont dépend la représentation d'un tel cadre. En revanche, j'assume pleinement le choix de ne pas avoir introduit l'analyse de la polyphonie, évoquée par Yocaris, car cela aurait apporté beaucoup de complexité au modèle, pour un rendement que je pense au final assez faible si l'on tente en l'occurrence de saisir la dynamique de la mise en intrigue. J'ai souvent abordé la question de la polyphonie et ses effets sur le lecteur, notamment dans L'œuvre du temps (voir les chapitres : «Ce que 
l'auteur fait à son lecteur (que son texte ne fait pas tout seul)", "La Fiction peut-elle mentir?», "Regarder le monde en face?») et dans plusieurs articles ultérieurs consacrés aux romans de Houellebecq (voir entre autres Baroni 2017b ; 2016 ; 2014 ; Baroni et Langevin 2016).

Si je produis un jour un ouvrage de synthèse plus général sur l'analyse des récits, nul doute que cet aspect, qui relève de la critique polyphonique, deviendra l'un des éléments centraux de la théorie, car il détermine dans une large mesure l'intérêt éthique et aléthique des fictions littéraires, tout en ouvrant l'analyse à la pluralité interprétative. En revanche, dans le périmètre plus restreint de mon dernier ouvrage, il me semblait que l'analyse de la polyphonie restait relativement neutre si l'on considère son effet direct sur la dynamique de l'intrigue, et qu'ouvrir cette boîte de Pandore aurait nui à l'objectif principal, qui était d'offrir un répertoire de concepts facilement mobilisables pour mettre en lumière les principales stratégies discursives présidant à la mise en intrigue.

Ilias Yocaris affirme que "l'expérience du terrain montre que la lecture "experte" est accessible à tous les élèves, pour peu qu'on dispose des moyens requis (et des compétences adéquates) pour les former correctement et que le contexte s'y prête ». Cela me réjouit, puisque c'est exactement ce genre de lecture que j'espère encourager avec les quelques outils que je passe en revue dons mon livre. Mais Yocaris ajoute ensuite une affirmation qui me semble contradictoire avec la précédente, puisqu'il soutient qu'il est impossible « de mettre en place une stylistique de la tension narrative pleinement intégrative, puisque cette opération requiert par définition des compétences techniques et des repérages langagiers inaccessibles à des nonspécialistes - et a fortiori des apprenants ».

Peut-on ou ne peut-on pas démonter les rouages de l'intrigue dans un contexte scolaire ? S'agit-il d'une approche réservée aux happy few de la critique académique ? Je n'arrive pas à comprendre la position de mon contradicteur. Pour ma part, je suis un optimiste. Ma propre expérience de terrain, dans un cours d'introduction à la littérature qui réunit des apprenant·e's allophones de niveau B1 et B2 aux parcours scolaires marqués par une forte hétérogénéité, me pousse à croire que c'est la première affirmation qui est la plus proche de la vérité. Lorsque je leur demande de réfléchir aux éléments qui renforcent la tension d'une scène dans une nouvelle de Maupassant, mon expérience montre que de nombreux apprenante-s sont capables de repérer des indices textuels pertinents, pour autant que l'on ait au préalable attiré leur attention sur le rythme du récit, les jeux de focalisation, la construction textuelle du point de vue ou l'effet de prolepses suggestives ou d'analepses ambiguës.

\section{L'intrigue comme « dynamisme intégrateur » ?}

Un autre aspect, qui me semble absolument devoir être défendu mais qui rencontrera certainement de nombreuses résistances, concerne l'extension du concept d'intrigue. Yocaris affirme en effet que :

l'intrigue est à ses yeux un dispositif textuel visant à intriguer le lecteur, définition axée (encore et toujours) sur la réception des récits fictifs. Or, ce choix méthodologique peut à bon droit sembler contestable, parce qu'il permet d'évacuer subrepticement une conception plus globalisante et plus synthétique de l'intrigue. 

une approche axée "sur la réception ", c'est plutôt la dissociation que je propose d'introduire entre l'intrigue comme dispositif intrigant et l'intrigue comme dispositif configurant, au sens de Paul Ricœur - ou plus exactement au sens du Paul Ricœur du premier tome de Temps et récit (1983). Ce que Yocaris peine à saisir, c'est que la différence entre nos deux approches n'est pas liée au fait que l'une, la mienne, serait fondée sur une analyse des effets, et l'autre, celle de Ricœur, sur une analyse du travail de configuration de l'auteur. Dans un cas comme dans l'autre, l'intrigue-intrigante et l'intrigue-configurante sont saisies dans la dynamique herméneutique d'un triple horizon : l'horizon intentionnel de l'auteur, l'horizon textuel de l'œuvre configurée, et l'horizon esthétique d'un lecteur confronté à ce texte. J'ai expliqué ailleurs (Baroni 2010 ; 2018) que la confusion entre intrigue et configuration - autrefois utile quand il s'agissait, dans un contexte constructiviste, de critiquer la fausse transparence des textes scientifiques - a fini par engendrer de nombreux problèmes et est devenue contre-productive, car elle ne rend pas justice à la spécificité de la mise en forme de l'expérience opérée par les récits que j’appelle mimétiques pour éviter de restreindre cette opération aux seuls récits fictionnels.

29 À la suite du philosophe américain Hayden White, Ricœur opère le rapprochement entre intrigue et configuration pour affirmer que les récits historiques et les fictions littéraires auraient pour fonction fondamentale de mettre en forme une expérience temporelle qu'il désigne, au début de sa longue méditation, comme "confuse, informe et, à la limite, muette » (1983:12). Comme j'ai essayé de le montrer à plusieurs reprises (Baroni 2009; 2010; 2018), Ricœur lui-même, dans la suite de sa réflexion, et notamment dans le tome 3 de Temps et récit (1985), finit par rejeter cette 
indifférenciation de principe entre la configuration du discours historiographique, qui vise à réinscrire le temps vécu dans le temps collectif des calendriers et des archives, et celle du discours fictionnel. Il affirme en effet que la fiction serait en fin de compte le lieu où l'expérience - qui serait déjà, à l'origine, concordante-discordante - pourrait être ressaisie dans sa complexité originelle, de sorte que seraient révélées les apories des solutions philosophiques au problème du temps. Il écrit en effet que :

[c]'est dans la littérature de fiction principalement que sont explorées les innombrables manières dont l'intentio et la distentio se combattent et s'accordent. En cela, cette littérature est l'instrument irremplaçable d'exploration de la concordance discordante que constitue la cohésion d'une vie. (1985 : 248-249)

30 Je trouve que ces différents états de la thèse de Ricœur sont certes passionnants d'un point de vue philosophique et herméneutique, mais leur champ d'application dans différents secteurs des sciences humaines, et en particulier dans l'analyse littéraire, a contribué à masquer une différence profonde entre des stratégies discursives et des styles narratifs qui apparaissent en définitive pratiquement opposés. On emploie aujourd'hui de manière indifférenciée cette idée d'une mise en intrigue comme « dynamisme intégrateur » (pour reprendre les termes de Yocaris) pour se référer à la structuration d'un article de presse, d'un reportage immersif, d'une biographie, d'un ouvrage historique, d'un roman de Robbe-Grillet ou de l'intrigue d'une série policière. Pour le dire aussi simplement que possible, s'il y a effectivement des représentations narratives dans lesquelles la visée du discours correspond à un objectif de mise en forme du réel - dans le but de rendre les événements plus compréhensibles, d'en augmenter la lisibilité ou la signifiance -, il me semble qu'il existe par ailleurs des mises en intrigue qui reposent sur une stratégie pratiquement opposée, et pour lesquelles la planification ou l'achèvement du discours ne joue pas un rôle central, ce qui explique que les intrigues prolifèrent dans les environnements sériels autant, voire même davantage, que dans les œuvres marquées par une forte unité.

31 Pour les récits mimétiques, le recours à la mise en intrigue viserait donc plutôt à replonger le destinataire au cœur de l'expérience racontée (qu'elle soit réelle ou fictive), ce qui contribue certes à établir une forme de compréhension profonde des événements, mais cette profondeur repose davantage sur des effets d'empathie, sur des dilemmes moraux inextricables et une complexification des perspectives que sur le dynamisme intégrateur d'un discours. On comprendra donc que c'est précisément parce que j'adopte une approche tenant compte des "paramètres liés à la stratégie discursive " qui président à la mise en forme des récits que cette distinction entre configuration et mise en intrigue devient centrale ${ }^{3}$.

\section{Focalisation et point de vue}

Je terminerai ce rapide tour d'horizon des critiques dont j'ai fait l'objet pour revenir sur une proposition qui nécessite probablement quelques clarifications et développements ultérieurs. Ilias Yocaris affirme que ma proposition d'utiliser conjointement l'analyse de la focalisation (qui fait l'objet d'une mise à jour terminologique et conceptuelle dans mon dernier livre) et l'analyse de la construction textuelle du point de vue apparaît

comme un compromis bancal entre l'approche genettienne (que l'on se refuse manifestement à abandonner tout à fait) et l'approche rabatélienne (que l'on ne parvient pas dès lors à exploiter pleinement). En effet, comme l'explique Baroni, la 
«focalisation restreinte " n'est rien d'autre qu'un avatar de la «focalisation externe » genettienne, la "focalisation élargie » venant se substituer, elle, à la «focalisation zéro ». Or, il est utile de rappeler qu'Alain Rabatel remet radicalement en question les concepts même de "focalisation zéro " et de "focalisation externe ", sur la base de critères strictement linguistiques dont il semble impossible de faire l'économie - du moins jusqu'à la preuve du contraire.

Certes, j'ai bien conscience que les quelques pages que je consacre à la focalisation et à la théorie de Rabatel sur le point de vue représenté mériteraient des développements supplémentaires. J'ai commencé à m'y mettre et deux articles paraîtront prochainement sur ce sujet, l'un dans une perspective didactique (2020), et l'autre dans le sillage du colloque d'hommage à Genette, qui m'a permis de revenir sur ces questions. Je reconnais bien volontiers l'importance majeure de cette longue filiation des modèles alternatifs à la focalisation genettienne, dont le but est de définir de manière beaucoup plus précise la construction des effets de subjectivité dans le récit. Cette tradition critique débute avec Mieke Bal $(1977 ; 1985)$ et se prolonge, entre autres, dans les travaux de Shlommith Rimmon-Kenan (1983), de François Jost (1989) ou de Manfred Jahn (1996), avant de trouver en France un aboutissement mieux ancré linguistiquement dans les travaux d'Alain Rabatel (voir notamment 1998 ; 2009).

Personnellement, je suis absolument convaincu que l'approche de Rabatel apporte des outils devenus incontournables pour étudier la manière dont le narrateur peut, occasionnellement, opérer un débrayage énonciatif (complet ou partiel) de sa perspective pour adopter (ou feindre d'adopter) le point de vue subjectif de tel ou tel personnage. Cette question du débrayage énonciatif est évidemment fondamentale, autant pour la dynamique de l'intrigue que pour l'étude de nombreux autres phénomènes, tels que les effets de polyphonie et l'orientation axiologique du discours. Je suis le premier à enseigner ces outils, qui remédient de manière bienvenue au manque d'ancrage linguistique de la plupart des approches narratologiques classiques.

Je crois toutefois nécessaire, lorsqu'on se penche sur les perspectives narratives, de distinguer clairement les questions relatives à la subjectivité représentée de celles que l'on pourrait qualifier de cybernétiques, c'est-à-dire qui renvoient strictement à la quantité d'information transmise au lecteur. Le désaccord profond entre Genette et les théories formelles de la subjectivité qui, depuis Mieke Bal jusqu'à Rabatel, s'intéressent aux personnages focalisateurs, repose sur cette distinction exposée par Burkhardt Niederhof, que je me permets de citer à nouveau, car il met le doigt sur un point fondamental :

Il y a de la place pour les deux [concepts] parce que chacun met en évidence un aspect différent d'un phénomène complexe et difficile à saisir. Le point de vue semble être la métaphore la plus efficace pour les récits qui tentent de rendre

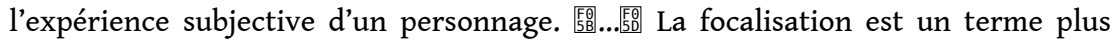
approprié lorsqu'on analyse la sélection des informations narratives qui ne servent pas à restituer l'expérience subjective d'un personnage mais à créer d'autres effets, tels que le suspense, le mystère, la perplexité, etc. (Niederhoff 2011 : \$18, m.t.)

Cela fait déjà plus de vingt ans que mon collègue stylisticien Gilles Philippe avait cerné le problème lorsqu'il affirmait que la «question fondamentale de la gestion de l'information dans le texte romanesque est généralement abordée à l'aide d'éclairantes mais encombrantes métaphores visuelles : point de vue, vision, focalisation, restriction de champ...» (1996: 81). Face au risque de confusion engendré par ces métaphores, Gilles Philippe insistait notamment sur la nécessité de distinguer clairement les paramètres textuels qui définissent la quantité de l'information de ceux qui concernent la 
qualité de l'information, où s'opposent les "textes à direction objective (accent mis sur le perçu) et les textes à direction subjective (accent mis sur le percevant)» $(1996: 82)$.

La nécessité de distinguer quantité et qualité de l'information m'a toujours parue évidente du fait mes collaborations interdisciplinaires - au sein du $\mathrm{GrEBD}^{4}$ ou du NaTrans ${ }^{5}$ - avec des membres de la section de cinéma de l'Université de Lausanne. Cela fait de nombreuses années que mon collègue Alain Boillat m'a rendu attentif au fait qu'à la suite des travaux de François Jost sur l'ocularisation et l'auricularisation (Jost 1987 ; Jost et Gaudreault 1990) les études filmiques considèrent comme banal de différencier l'ancrage subjectif de la représentation audio-visuelle de la question plus générale de la gestion des savoirs transmis aux spectateurs, ce dernier aspect déterminant la dynamique de l'intrigue, ainsi que le soulignent les travaux de David Bordwell ${ }^{6}$. Cela ne veut pas dire que ces deux manières d'aborder la question des perspectives narratives seraient sans rapports l'une avec l'autre, mais il s'agit de deux aspects différents renvoyant à un phénomène complexe. L'innovation que je propose dans mon dernier livre est donc toute relative si l'on se place dans une perspective transmédiale, puisqu'elle fait écho à des approches bien établies dans d'autres disciplines (cf. Baroni 2017c).

D'ailleurs, je mentionne le cas de la scène d'ouverture des Dents de la mer (1975) dans laquelle le récit est focalisé sur une baigneuse qui se fera dévorer par un requin. L'usage de la caméra subjective, qui montre la baigneuse en contre-plongée (c'est le cas de le dire) du point de vue sous-marin du prédateur, est un moyen de signaler la présence du requin et de renforcer le suspense. L'ocularisation interne produit donc une focalisation élargie plutôt qu'elle ne débouche sur une restriction de champ : le public tremble pour la baigneuse insouciante, parce qu'il en sait davantage que cette dernière, qui ignore le danger imminent qui la menace. Si l'on ne distingue pas point de vue et focalisation, on déboucherait ainsi sur une contradiction, puisqu'une focalisation « interne » (point de vue du requin) serait en même temps une focalisation « zéro » (le réalisateur nous en montre plus que ce que peut connaitre le personnage). J'ajouterai que l'analyse de l'ocularisation au cinéma - qui est très proche, dans son principe sinon dans ses moyens formels, de l'analyse de la construction textuelle du point de vue - est un élément clé pour l'analyse de la dynamique de l'intrigue, mais que son interprétation passe par le recours à la prise en compte d'une autre dimension : celle de l'analyse des savoirs mis en scène par la représentation, où la mesure passe par la comparaison entre les connaissances du public et ce que savent les personnages focalisé par le récit.

Pour expliciter la chose, je prendrai un autre exemple, littéraire cette fois, tiré d'une nouvelle de Maupassant. L'Auberge raconte l'histoire d'Ulrich Kunzi, qui s'occupe du gardiennage hivernal d'une auberge de montagne avec son compagnon Gaspard Hari et leur chien Sam. Après la disparition et la mort probable de Gaspard pendant une nuit de tempête, la culpabilité, la solitude et l'excès d'alcool font tomber Ulrich dans la folie, et il finit par s'imaginer entendre les cris de son ami disparu, comme s'il revenait le hanter. Au moment du climax de ce court récit (dont la nature conclusive est signalée par l'expression " une nuit enfin »), Ulrich ouvre la porte de l'auberge pour confronter le spectre:

Une nuit enfin, pareil aux lâches poussés à bout, il se précipita sur la porte et l'ouvrit, pour voir celui qui l'appelait et pour le forcer à se taire.

Il reçut en plein visage un souffle d'air froid qui le glaça jusqu'aux os et il referma le battant et poussa les verrous, sans remarquer que Sam s'était élancé dehors. Puis, 
frémissant, il jeta du bois au feu pour se chauffer; mais soudain il tressaillit, quelqu'un grattait le mur en pleurant.

Il cria éperdu : «Va-t'en. » Une plainte lui répondit, longue et douloureuse.

Alors tout ce qui lui restait de raison fut emporté par la terreur. Il répétait «Vat'en » en tournant sur lui-même pour trouver un coin où se cacher. L'autre, pleurant toujours, passait le long de la maison en se frottant contre le mur. (Maupassant $1880: 136$ ) l'état émotionnel d'Ulrich, sur ses perceptions et ses pensées. Le texte n'est pas intégralement en point de vue interne, mais il adopte majoritairement cette perspective subjective pour renforcer le pathos de la scène et nous faire ressentir la terreur éprouvée par le protagoniste. En même temps, le narrateur nous en dit plus que ce que sait le personnage, puisqu'il nous signale qu'Ulrich ne remarque pas que «Sam s'était élancé dehors ». Cette information cruciale nous permet de comprendre que ce sont les efforts du chien pour rejoindre son maître qui sont à l'origine de la terreur éprouvée par Ulrich, ce qui renforce non seulement le suspense mais aussi l'ironie tragique de la situation. On voudrait apostropher Ulrich et lui faire reprendre ses esprits, lui dire d'ouvrir la porte pour sauver son chien et recouvrer sa raison, tout comme on aurait aimé crier à la nageuse de rejoindre le rivage. Mais le processus de folie est trop avancé, ainsi que nous en informe le narrateur, qui nous place, jusqu'à la fin de la séquence, dans le point de vue du personnage. Dans cette perspective bornée, le chien n'est jamais identifiable en tant que tel, le texte nous le désigne comme « quelqu'un », « Une plainte », « l'autre ».

Dans ce cas, comme dans la scène des Dents de la mer, l'approche genettienne classique déboucherait sur une contradiction entre focalisation «interne» et focalisation «zéro ». Le paradoxe pourrait être résolu en recourant au bricolage conceptuel que constitue ce que Genette appelait une altération du mode, qui renverrait en l'occurrence à la figure de la paralepse. À mes yeux, il n'y a pas d'altération du mode : le narrateur (ou l'auteur) nous dit strictement ce qu'il veut, il est libre d'adopter occasionnellement le point de vue du personnage ou de s'en écarter quand cela s'avère nécessaire pour produire tel ou tel effet. Parler alors de focalisation «zéro" ou « variable » me semble trop vague et ne rend pas justice à la complexité du dispositif. Il vaut mieux, alors, recourir à la perspective offerte par Alain Rabatel pour montrer les éléments textuels qui nous placent dans le point de vue interne du personnage, tout en expliquant que la focalisation est, à cet endroit, stratégiquement élargie, de manière à renforcer le suspense de la scène. Le dispositif repose ainsi sur la combinaison entre un rapport empathique avec le personnage et un savoir augmenté par rapport à sa perspective cognitive limitée.

Il reste certainement de nombreuses précisions à apporter à cette reformulation de la focalisation et il faudrait mieux articuler cette dernière avec la théorie linguistique du point de vue, mais je suis convaincu qu'elle règle de nombreux problèmes inhérents à la théorie de la focalisation telle qu'elle est enseignée depuis des décennies. Depuis que j'explique en classe la différence entre point de vue et focalisation, et comment combiner ces deux approches pour interpréter la dynamique des textes littéraires (ou celle des planches de bande dessinée), la très grande majorité des étudiant·es appréhendent ces outils avec aisance et les mobilisent sans difficulté pour étayer leurs interprétations. Quand je compare cela avec les définitions incroyablement floues de la focalisation que 
l'on trouve, encore aujourd'hui, dans différents ouvrages de synthèse ${ }^{7}$, je me dis qu'il y a vraiment un gain heuristique à procéder de la sorte.

Certes, s'il fallait vraiment ne garder qu'un seul modèle, je pencherais pour l'approche de Rabatel, qui a l'avantage de cerner de manière beaucoup plus précise le fonctionnement des «textes à orientation subjective». Mais je trouverais regrettable de ne plus pouvoir recourir à des concepts supplémentaires permettant de s'interroger sur l'alternance de différents foyers narratifs (le personnage focalisé par le récit n'étant pas nécessairement saisi de l'intérieur $\left.{ }^{8}\right)$ ou sur des cas marqués où l'auteur joue sur une restriction ou un élargissement du champ de savoirs auxquels les lecteurs peuvent accéder.

Quant à cette démonstration évoquée par Ilias Yocaris, dans laquelle Rabatel (1997) exclut l'existence de la focalisation externe, elle résulte selon moi d'un malentendu, même si je reconnais que son origine est largement imputable à Genette. Comme j'ai essayé de l'expliquer, il ne s'agit pas de prouver que Rabatel a tort ou que Genette a raison. Quand on se place dans une interprétation de la focalisation sous l'angle la qualité de l'information, Rabatel a certainement raison d'affirmer que l'origine d'un énoncé ne peut être rattachée qu'à deux instances possibles: le narrateur ${ }^{9}$ ou le personnage. Mais, si la définition de la focalisation repose strictement sur une approche quantitative, qui consiste à comparer ce que savent lecteurs à ce que savent personnages, alors la question est complètement différente, et il faut bien tenir compte de trois possibilités : l'égalité, la supériorité ou l'infériorité.

Je disais que le malentendu est imputable à Genette, car cette orientation strictement quantitative n'est pas toujours très claire dans les définitions qu'il donne de la focalisation, qui oscillent entre des considérations sur la subjectivité et d'autres sur la restriction de champ. La conception cybernétique apparaît plus clairement dans l'une des sources dont s'inspire Genette: Tzvetan Todorov (1966:141-142) représente ces rapports par des symboles mathématiques $(=,<,>)$ au lieu de recourir aux étiquettes interne, externe et zéro. L'usage des termes restreint et élargi vise simplement à retrouver ce modèle quantitatif fondé sur une comparaison entre des valeurs sans devoir nécessairement poser à chaque fois le comparant (ce que sait le lecteur) et le comparé (ce que sait le personnage focalisé).

Genette a probablement pris conscience du problème assez tardivement, lorsqu'il se voit reprocher de ne pas avoir tenu compte du personnage comme focalisateur potentiel du récit ${ }^{10}$. Dans Nouveau discours du récit, il précise :

Par focalisation, j'entends donc bien une restriction de "champ ", c'est-à-dire en fait une sélection de l'information narrative par rapport à ce que la tradition nommait l'omniscience. (Genette 2007 : 348)

Et il ajoute :

Pour moi, il n'y a pas de personnage focalisant ou focalisé : focalisé ne peut s'appliquer qu'au récit lui-même, et focalisateur, s'il s'appliquait à quelqu'un, ce ne pourrait être qu'à celui qui focalise le récit, c'est-à-dire le narrateur - ou, si l'on veut sortir des conventions de la fiction, l'auteur lui-même, qui délègue (ou non) au narrateur son pouvoir de focaliser, ou non. (Genette 2007 : 347)

Genette affirmait que la recherche n'est "qu'une série de questions » et il ajoutait que l'essentiel était « de ne pas se tromper de question» (2007: 350). Il me semble que les antagonistes auraient pu se réconcilier s'ils s'étaient accordés sur le fait que leurs réponses divergentes ne portent tout simplement pas sur les mêmes questions. Rabatel 
a raison d'affirmer que le point de vue ne peut être orienté que selon deux axes : celui $\mathrm{du}$ narrateur ou celui du personnage, et Genette a eu tort de parler de focalisation « interne » ou « externe ». Mais l'approche genettienne (et avec lui, celles de Todorov, Pouillon, Blain, Lubbock et quelques autres) qui consiste à s'intéresser à la sélection de l'information narrative par rapport à ce que la tradition nommait l'omniscience demeure certainement une piste valide, ou du moins utile, car elle éclaire certains paramètres fondamentaux pour la dynamique de l'intrigue.

\section{BIBLIOGRAPHY}

Adam, Jean-Michel (2005), La linguistique textuelle. Introduction à l'analyse textuelle des discours, Paris, Armand Colin.

Bal, Mieke (1977), Narratologie. Essais sur la signification narrative dans quatre romans modernes, Paris, Klincksieck.

Bal, Mieke (2009 宧1985塸), Narratology. Introduction to the Theory of Narrative, Toronto, Buffalo \& London, University of Toronto Press.

Baroni, Raphaël (à paraître en 2020), « Pour des concepts narratologiques intelligibles et utiles pour l'enseignement : schéma quinaire et focalisation en débat », Transpositio, $\mathrm{n}^{\circ} 2$.

Baroni, Raphaël (2018), « Face à l'horreur du Bataclan : récit informatif, récit immersif et récit immergé », Questions de communication, $\mathrm{n}^{\circ} 34$, p. 107-132.

Baroni, Raphaël (2017a), Les Rouages de l'intrigue. Les outils de la narratologie postclassique pour l'analyse des textes littéraires, Genève, Slatkine érudition.

Baroni, Raphaël (2017b) « Combien d'auteurs y a-t-il dans cette œuvre ? », Fabula / Les colloques, Les « voix » de Michel Houellebecq, URL : http://www.fabula.org/colloques/document4222.php

Baroni, Raphaël (2017c) « Pour une narratologie transmédiale », Poétique, n 182, p. 155-175. URL : https://www.cairn.info/revue-poetique-2017-2-p-155.htm

Baroni, Raphaël (2016) « Comment débusquer la voix d'un auteur dans sa fiction. Une étude de quelques provocations de Michel Houellebecq ", Arborescence, n 6, p. 72-93.

Baroni, Raphaël (2014) «La guerre des voix : critique polyphonique et divergences interprétatives dans l'œuvre de Michel Houellebecq ", COnTEXTE, Varia.

URL : http://contextes.revues.org/5979

Baroni, Raphaël (2010), «Ce que l'intrigue ajoute au temps. Une relecture critique de Temps et récit de Paul Ricœur », Poétique, n 163, p. 361-382.

Baroni, Raphaël (2009), L'Écuvre du temps. Poétique de la discordance narrative, Paris, Seuil.

Baroni, Raphaël (2007), La Tension narrative. Suspense, curiosité, surprise, Paris, Seuil.

Baroni, Raphaël \& Francis Langevin (dir.) (2016), « Polyphonies : voix et valeurs du discours littéraire ", Arborescence, n 6. URL : https://www.erudit.org/revue/arbo/2016/v/n6/index.html Barthes, Roland (1970), S/Z, Paris, Seuil. 
Bemporad, Chiara (2014), « Lectures et plaisirs : pour une reconceptualisation des modes et des types de lecture littéraire ", Etudes de Lettres, n² 295, p. 65-83.

Bordwell, David (1985), Narration in the Fiction Film, Madison, University of Wisconsin Press. Bourdieu, Pierre (1971), « Le Marché des biens symboliques », L'année sociologique, n 22 p. 49-106. Daunay, Bertrand (2018), « Recension : Raphaël Baroni (2019), "Les rouages de l'intrigue” », Transpositio, Conversations critiques, URL : http://www.transpositio.org/articles/view/recensionbaroni-raphael-2017-les-rouages-de-l-intrigue

Daunay, Bertrand (1999), « La "lecture littéraire" : les risques d'une mystification », Recherches, n³0, p.29-59.

Gabathuler, Chloé (2016), Apprécier la littérature. La relation esthétique dans l'enseignement des textes littéraires, Rennes, PUR.

Genette, Gérard (2007), Discours du récit, Paris, Seuil, coll. « Points »

Jahn, Manfred (1996), « Windows of Focalization : Deconstructing and Reconstructing a Narratological Concept », Style, n³0, p. 241-267.

Maingueneau, Dominique (2004), Le Discours littéraire. Paratopie et scène d'énonciation, Paris, Armand Colin.

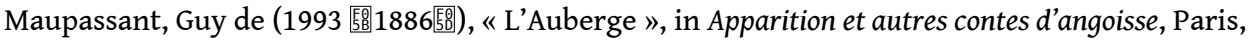
GF Flammarion.

Niederhoff, Burkhard (2011), « Focalization », The Living Handbook of Narratology. Consulté le 15 janvier 2018. URL : http://www.lhn.uni-hamburg.de/article/focalization

Jost, François (1989 [1987]), L'Cil-caméra. Entre film et roman, Lyon, PUL.

Jost, François \& André Gaudreault (2017 [1990]), Le Récit cinématographique, Films et séries télévisées, Armand Colin Cinéma, Paris, Armand Colin, $3^{\mathrm{e}}$ édition.

Jouve, Vincent (2019), Les Pouvoirs de la fiction. Pourquoi aime-t-on les histoires ?, Paris, Armand Colin.

Jouve, Vincent (1992), L'Effet-personnage dans le roman, Paris, PUF.

Merminos, Gilles (2019), Histoire d'une nouvelle. Pratiques narratives en salle de rédaction, Bruxelles, De Boeck Supérieur.

Patron, Sylvie (2009), Le Narrateur, Paris, Armand Colin.

Philippe, Gilles (1997), Le Roman. Des théories aux analyses, Paris, Seuil, coll. « Memo ».

Rabatel, Alain (2009), Homo Narrans, Limoges, Editions Lambert-Lucas, deux volumes.

Rabatel, Alain (1998), La Construction textuelle du point de vue, Lausanne et Paris, Delachaux et Niestlé.

Rabatel, Alain (1997), «L'introuvable focalisation externe. De la subordination de la vision externe au point de vue du personnage ou au point de vue du narrateur ", Littérature, $\mathrm{n}^{\circ} 107 \mathrm{p}$. 88-113.

Reuter, Yves (2016), L'analyse du récit, Paris, Armand Colin, coll. « $128 », 3^{\mathrm{e}}$ édition.

Ricœur, Paul (1983), Temps et récit. 1. L'intrigue et le récit historique, Paris, Seuil, coll. « Points ». Ricœur, Paul (1985), Temps et récit. 3. Le temps raconté, Paris, Seuil, coll. « Points ». 
Rimmon-Kenan, Shlomith (2002 㗨1983嚂), Narrative Fiction, Londres \& New York, Routledge.

Ronveaux, Christophe, Bernard Schneuwly \& GRAFElitt (2019), « Discipliner par la littérature », in Approches didactiques de la littérature, N. Denizot, J.-L. Dufays \& B. Louichon (dir.), Namur, PUN, p. 139-156.

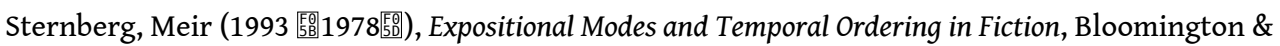
Indianapolis, Indiana University Press.

Todorov, Tzvetan (2007), La Littérature en péril, Paris, Flammarion.

Todorov, Tzvetan (1966), « Les catégories du récit littéraire », Communication, n 8, p. 125-151.

Vanoost, Maire (2016), « Journalisme narratif : des enjeux contextuels à la poétique du récit », Cahiers de narratologie, $\mathrm{n}^{\circ} 3$. Consulté le 10 décembre 2019. URL : https://

journals.openedition.org/narratologie/7543

Wagner, Frank (2011), « Raconter est devenu proprement impossible. Fabula et intrigue dans l'œuvre romanesque d'Alain Robbe-Grillet ", Atelier de théorie littéraire. Fabula. Consulté le 24 août 2014. URL : http://www.fabula.org/atelier.php?Fabula_et_intrigue

Wagner, Frank \& Francine Dugast-Portes (dir.) (2010), Lectures de Robbe-Grillet (Les Gommes, La Jalousie), Rennes, PUR.

\section{NOTES}

1. Sur le caractère cyclique de cette crise et sur son contexte actuel, je renvoie au premier numéro de la revue Transpositio : http://www.transpositio.org/

2. Je ne reviens pas sur la critique de Yocaris concernant ma lecture des Gommes de Robbe-Grillet. Il est évident que l'approche proposée dans la partie 3 visait avant tout à montrer que des mécanismes présidant au nouement et au dénouement d'une intrigue restent parfaitement lisibles dans la plupart des chefs-d'œuvre de la modernité du siècle passé, ce qui explique la surprise de ce «bonheur de lecture» qui découle «de l'emprise fascinante exercée par un univers fictionnel d'une remarquable densité » qu'évoque Frank Wagner lors de sa première réception des Gommes (Wagner 2011). Il ne s'agissait donc pas d'expliquer l'esthétique du Nouveau Roman par le biais d'un commentaire sur l'intrigue, mais de montrer que cette révolution esthétique ne remet pas complètement en cause la dynamique intrigante, puisqu'elle détourne autant qu'elle exploite les stéréotypes du roman policier et d'espionnage. À nouveau, il s'agissait de dépasser l'opposition traditionnelle entre commercialité et valeur esthétique, de manière à mettre en question l'affirmation péremptoire que les grandes œuvres de la modernité seraient nécessairement dépourvues d'intrigue. Pour une approche plus compréhensive de cette œuvre très riche, je renvoie à d'autres ouvrages plus complets, par exemple (Wagner \& DugastPortes 2010).

3. L'analyse comparée du traitement journalistique des attentats du 13 novembre 2015 que j'ai récemment publiée dans la revue Questions de communication (Baroni 2018) permet de saisir l'importance, également dans le domaine de l'infocom, de distinguer un journalisme immersif, qui se plie aux contraintes de la mise en intrigue, d'un journalisme d'information, qui vise au contraire à inscrire les événements traumatiques dans une configuration qui en rend l'interprétation possible. Le choix journalistique de la mise en intrigue, qui devient de plus en plus banal avec le développement du journalisme narratif et de l'infotainment, possède ses risques et ses vertus, mais on ne peut le comprendre que si on l'oppose à une autre manière de raconter les événements de l'actualité. Voir également Merminod (2019) et Vanoost (2016).

4. Groupe d'étude sur la bande dessinée : http://wp.unil.ch/grebd/ 
5. Pôle de narratologie transmédiale : https://wp.unil.ch/narratologie/natrans/

6. Il faut noter que Bordwell (1985) s'inspire des travaux de Meir Sternberg (1978) sur les intérêts narratifs.

7. Sur cette question, voir Baroni (2020). Voir notamment la dernière édition de l'ouvrage d'Yves Reuter (2016), dont l'indécision entre les approches de Genette et de Rabatel pour définir la focalisation rend l'appréhension de cette dernière totalement obscure.

8. Certes, Rabatel (2009) parle alors de point de vue « embryonnaire », mais l'absence de marques formelles pour déterminer de tels foyers, en dehors de la quantité des informations accumulées, me semble s'écarter de la perspective proprement linguistique qui est la sienne.

9. Ici, je modalise quand même, car d'autres approches linguistiques, dans lesquelles Sylvie Patron (2009) s'inscrit, mettent en cause, avec des arguments très convaincants, la présence nécessaire d'un narrateur pour le récit de fiction. Il faudrait donc tenir compte de cas où le récit n'est orienté ni par le personnage, ni par la figure d'un narrateur optionnel, ce qui a été thématisé par l'idée d'un centre déictique vide.

10. Notons également que Genette, à cette époque, a pris connaissance des travaux de François Jost, et il affirme ceci : "Sur la différence entre focalisation et "ocularisation" (information et perception), et sur l'intérêt de cette distinction pour la technique du film et celle du Nouveau Roman, voir Jost, 1983a et 1983b chap. III ("La mobilité narrative"). Remontant de ces cas limites vers le régime ordinaire du récit, le travail de Jost me semble la contribution la plus pertinente au débat sur la focalisation, et à l'affinement nécessaire de cette notion » (Genette 2007 : 348).

\section{AUTHOR}

\section{RAPHAËL BARONI}

Université de Lausanne 